sixteen papers, one statistical, being on the work of the hospital attached to the association. There are two interesting papers, one on the motility of intra-ocular cysticercus based on a study of eleven cases, and the second a long paper on ocular oncocerciasis. Other papers are on quinine blindness; retinal detachment, the latter on the technique and value of hypertonic injections into Tenon's capsule ; squint ; orthoptics ; anaesthesia in operations for cataract ; contact lenses; tuberculosis of the choroid; angio-scotometry; pterygium as well as other papers.

Each paper is furnished with an abstract in English at the end. Paper and printing are good and it is a pity that some of the microphotographs and fundus photographs have not come out better.

\title{
CORRESPONDENCE
}

\section{LACHESINE, A NEW MYDRIATIC}

To the Editors of THE BRITISH JOURNAL OF OPHTHALMOLOGY.

DEAR SIRS, - In a recent issue of your Journal, Professor W. J. B. Riddell ${ }^{1}$ and Professor Ida Mann $^{2}$ reported their independent observations on the clinical use of a new mydriatic, benzilyloxyethyl dimethylethylammonium chloride, which has been known up till now as E3. May-I announce that E3 has now been given the name Lachesine (from $\lambda \alpha \dot{\alpha} \epsilon \sigma \iota s$ one of the Fates whose

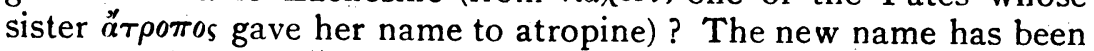
approved by the Director of Research and Development of the Ministry of Supply, who permits me to say that the Ministry's stock of the drug is being distributed by Messrs. Whiffen and Sons Ltd. (of Carnforth Road, Fulham, S.W.6) to whom all enquiries for supplies of Lachesine should be made.

$$
\text { I am, Sirs, }
$$

\section{Yours truly,}

1. Brit. Jl. Ophthal., Vol. XXX, p. 1, 1946.

$$
\text { H. R. ING. }
$$

\section{Vol. XXX, p. 8, 1946.}

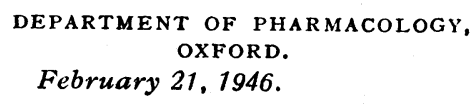

To the Editors of The BRITISH Journal of OPHThalmology.

DEAR SIRS,-I was interested in Professor Milroy Paul's article in your January issue, not only on account of its content but also 
because it called up various trains of thought more or less related to his report. Some of these I have jotted down and take the liberty of forwarding.

The impression is given that the choice of the Shugrue operation was largely determined by the greater liability of the Krönlein incision to damage the facial nerve fibres, although " recorded cases make no mention of paralysis of the orbicularis oculi muscles after the operation." Surely the fact is that the classical Krönlein incision is not sufficiently damaging to be followed ordinarily by significant orbicularis paralysis. This is not really so surprising when one considers the anatomical distribution. I am not in a position to comb the many German and French references which bear on the subject ; but that orbicularis paralysis may be a sequel is acknowledged by Birsch-Hirschfeld in Elschnig's Augenarztliche Operationslehre (1922 Ed.), although he had not experienced it in 39 cases of his own. This led me to think of what the average ophthalmologist means by "recorded cases" and "the literature." I imagine we risk being wide of the mark unless we qualify these expressions. Few of us have either the time, inclination or ability to make an exhaustive search of the literature. With the help of the Royal Society of Medicine, the Surgeon General's Catalogue and the Cumulative Index however, it is possible to cover all the more widely circulated material, and many of the minor and obscure records. Such searches are often more academic than practical, since the common facts of our art are readily available in text books and obscure observations of good quality tend eventually to come to the surface or be re-discovered. Few of us are likely to quarrel with Professor Paul when he says " the removal of a retrobulbar intra-orbital tumour is a rare event," at all events it is relatively so, especially in the sense he means, viz., removal with conservation of the globe and partial visual function.

The frequency of intra-orbital tumours appears to vary in different parts of the world, and in some places the angiomata are not uncommon relative to other intra-orbital tumours, excluding those extending from the globe. The true encapsulated cavernous angioma may perhaps be regarded as the ophthalmic surgeon's good dream in this field, unless indeed the fronto-ethmoidal mucocele is allowed to compete. This does not apply to a cavernous angioma within the muscle cone, although it is perhaps the least objectionable tumour to find in this situation. I have had the opportunity of exploring large numbers of intra-orbital tumours and removing many of them. I have only once employed the Krönlein approach, rather as an experimental measure and not for a tumour within the muscle cone. Since accurate private records are not readily available I can only be sure of having removed one cavernous 
angioma situated truly within the muscle cone, and this by superoexternal orbitotomy. There were various other retrobulbar tumours removed by this route, however, and numbers of intra-orbital explorations in the 1 o'clock to 6 o'clock area of the orbital cone. The incision, following the curve of the orbital rim and situated just behind it, ordinarily swept from 1 o'clock to 4 o'clock. This curve does not affect so many facial nerve twigs as the reverse Krönlein curve. It does not appear to give rise to subsequent orbicularis trouble. I have never discussed technique in print or the approach adopted in the various quadrants, but my methods have been so akin to Benedict's in principle that the quotation from him by Spaeth gives a good idea of the approaches which I have used for many years. I am tempted to refer here to certain points in technique not stressed in our surgical text books. The advisability of timely tarsorrhaphy should be borne in mind in certain circumstances. I have known it preserve the sight for years while the globes slowly left the orbits and the skin of the lids became unbelievably thin. Iodoform powder, preferably precipitated and sterilized, freely applied with tampons, is most helpful in dealing with soft tissue ooze, just as wax in the case of bone. The use of the hammer, gouge and nibbling forceps, if and as required, makes for ease. It is surprising what extra access is given by a small amount of external marginal resection. The ophthalmic literature, in places, is apt to decry the chisel and endorse the dental engine and such like. My prediliction for the hammer and chisel was developed in early days by practical instruction at the hands of Alexander and Neuman, masters of craft in the Pollitzer tradition.

In this connection one's thought's turn to overlapping surgical fields about the orbit. It is perhaps not sufficiently common to find the ophthalmic surgeon well versed in the arts of his colleagues the neuro-surgeon and the E. N. T. surgeon. Practical training, especially in E. N. T. work, makes for greater peace of mind, if, when following up an orbital exploration one exposes part of the nasal cavity or the dura. It is very satisfactory to have such colleagues to co-operate with or even to hand over to in difficult circumstances, but hardly excusable to be other than familiar with their methods if even remotely applicable to our special field. One can hardly imagine a Czermak or an Elschnig hesitating to proceed centrifugally from the orbit across the boundary in any direction demanded by the occasion. On the other hand one welcomes those advances of keen and adventurous colleagues, who ordinarily work in adjoining territories, which enlarge our horizon to the patient's benefit.

These musings are not in any sense intended to detract from Professor Paul's record of a successful retrobulbar operation by an unusual method, on which he is to be congratulated. On the 
contrary, were I again situated where the intra-orbital problem was a commonplace, I should like to undertake a Shugrue-Moran approach for the sake of comparison, especially if decompression were an influential factor.

Yours faithfully,

R. E. WRIGHT.

FLEET, HANTS.

February 24, 1946.

\section{LATERAL ORBITOTOMY (Krönlein's Operation)}

To the Editors of THE BRITISH JOURNAL OF OPHTHALMOLOGY.

DEAR SIRS,-Professor Milroy Paul in his paper "Cavernous Haemangioma of the orbit successfully removed by Shugrue's operation," Brit. Jl. Ophthal., January, 1946, advises Shugrue's incision for lateral orbitotomy on the grounds that the other classical incisions are liable to cause orbicularis weakness through damage to branches of the seventh nerve.

I cannot claim any greater personal experience of Krönlein's operation than 7 cases, 2 of which were for the removal of a tumour inside the cone of the recti muscles and 5 for intra-orbital foreign bodies. In 5 of them I used a curved incision concentric with the lateral orbital margin from the centre of which a horizontal incision ran posteriorly about the level of the outer canthus over the malar bone and along the upper edgel of the zygoma. The 2 flaps thus fashioned by these incisions were undermined at the level of the periosteum of the malar-bone and the temporal fascia. In the two other cases 3 incisions made a flap with its base temporally.

In none of these cases was there any residual weakness of the orbicularis appreciated by clinical examination.

I think that the cleanest and least traumatic way of removing the lateral orbital wall is by either Gigli's or Stilli's saw. After the orbital periosteum is carefully separated from the bone it is retracted medially by a flat malleable retractor which serves also to protect the orbital contents. The temporal muscle is also retracted from the frontal process of the malar bone and the loop of one end of Gigli's saw is passed through the anterior extremity of the inferior orbital fissure and the bone cut in the horizontal plane of the lower orbital margin. On a horizontal plane a little above the external angular process and posteriorly at a point a little below where the temporal fossa merges with the base of the middle fossa of the skull a hole is made with an Archimedes drill, fitted with a stop, of sufficient size to allow the passage of the looped end of Gigli's saw. 